\title{
Marstrand's Theorem revisited: projecting sets of dimension zero
}

\author{
Victor Beresnevich* Kenneth Falconer Sanju Velani ${ }^{\dagger}$ \\ $\begin{array}{lll}\text { (YORK) } & \text { (ST ANDREWs) }\end{array}$
}

Agamemnon Zafeiropoulos

(YORK)

\author{
With an Appendix: The gap of uncertainty \\ David Simmons ${ }^{\S} \quad$ Han Yu Agamemnon Zafeiropoulos \\ $\begin{array}{lll}\text { (York) } & \text { (St ANDREWS })\end{array}$
}

\begin{abstract}
We establish a refinement of Marstrand's projection theorem for Hausdorff dimension functions finer than the usual power functions, including an analogue of Marstrand's Theorem for logarithmic Hausdorff dimension.
\end{abstract}

2010 Mathematics Subject classification: 28A80, 28A78, 11J83

\section{Introduction}

\subsection{Motivation}

Given $0 \leq \theta<\pi$, let $L_{\theta}$ denote the line through the origin of $\mathbb{R}^{2}$ that forms an angle $\theta$ with the horizontal axis. Let $\operatorname{proj}_{\theta}$ denote orthogonal projection onto the

\footnotetext{
${ }^{*}$ Research partly supported by EPSRC grant EP/J018260/1

${ }^{\dagger}$ Research partly supported by EPSRC grant EP/J018260/1

${ }^{\ddagger}$ Research supported by EPSRC Doctoral and Departmental Teaching Studentships

${ }^{\S}$ Research supported by EPSRC grant EP/J018260/1
} 
line $L_{\theta}$ and $\operatorname{dim} A$ denote the Hausdorff dimension of a set $A \subseteq \mathbb{R}^{2}$. Then $\operatorname{proj}_{\theta}$ is a Lipschitz mapping; indeed for all $\theta$,

$$
\left|\operatorname{proj}_{\theta} x-\operatorname{proj}_{\theta} y\right| \leq|x-y| \quad \forall x, y \in \mathbb{R}^{2} .
$$

This together with the trivial fact that $\operatorname{proj}_{\theta} A$ is a subset of a line, implies

$$
\operatorname{dim} \operatorname{proj}_{\theta} A \leq \min \{1, \operatorname{dim} A\},
$$

see for example [6, Proposition 3.3]. The famous projection theorem of Marstrand [10], dating from 1954, tells us that equality holds in (2) for almost almost all directions $\theta$ with respect to Lebesgue measure $\mathcal{L}$. Equivalently, the exceptional values of $\theta \in[0, \pi)$ for which the inequality (2) is strict, form a set of onedimensional Lebesgue measure zero.

Theorem (Marstrand). Let $A \subseteq \mathbb{R}^{2}$ be a Borel set.

(i) If $\operatorname{dim} A \leq 1$ then $\operatorname{dim} \operatorname{proj}_{\theta} A=\operatorname{dim} A$ for almost all $\theta \in[0, \pi)$.

(ii) If $\operatorname{dim} A>1$ then $\mathcal{L}\left(\operatorname{proj}_{\theta} A\right)>0$ for almost all $\theta \in[0, \pi)$.

Observe that the measure conclusion of (ii) is significantly stronger than the corresponding dimension statement; it trivially implies that $\operatorname{dim}_{\operatorname{proj}} A=1$ for almost all $\theta \in[0, \pi)$. Marstrand's proof depends heavily on delicate and, in places, complicated geometric and measure theoretic arguments. Subsequently, Kaufman [9] gave a slick, two page, proof that made natural use of the potential theoretic characterization of Hausdorff dimension and Fourier transform methods.

Here we will be concerned with the case when $\operatorname{dim} A \leq 1$. Indeed, to motivate our investigation, consider the extreme situation when $\operatorname{dim} A=0$. Then Marstrand's Theorem implies no more than the trivial statement that $\operatorname{dim} \operatorname{proj}_{\theta} A=0$ for all $\theta$. Thus, to obtain non-trivial information in such situations, it is natural to ask whether a version of Marstrand's Theorem remains valid for finer notions of Hausdorff dimension. One consequence of Theorem 2, our main result, is the following analogue of Marstrand's Theorem for logarithmic Hausdorff dimension, that is where the Hausdorff measures are defined with respect to dimension or gauge functions $(-\log r)^{-s}$ (for small $r$ ) and $s \geq 0$, and $\operatorname{dim}_{\log } A$ is the critical value of $s$ at which these measures jump from $\infty$ to 0 , see $\$ 1.2 .1$ for the full definitions.

Theorem 1. Let $A \subseteq \mathbb{R}^{2}$ be a Borel set. Then

(i) $\operatorname{dim}_{\log } \operatorname{proj}_{\theta} A \leq \operatorname{dim}_{\log } A$ for all $\theta \in[0, \pi)$,

(ii) $\operatorname{dim}_{\log } \operatorname{proj}_{\theta} A=\operatorname{dim}_{\log } A$ for $\mathcal{L}$-almost all $\theta \in[0, \pi)$. 
Remark 1. By considering the size of sets of exceptional angles, see §3․ㅡ, we are further able to conclude that

$$
\operatorname{dim}_{\log }\left\{\theta \in[0, \pi): \operatorname{dim}_{\log } \operatorname{proj}_{\theta} A<\operatorname{dim}_{\log } A\right\} \leq \operatorname{dim}_{\log } A .
$$

Of course, the interesting case is when $\operatorname{dim}_{\log } A$ is finite. Then, by definition $\operatorname{dim} A=0$ and so (3) is significantly stronger than Theorem 1 .

Before moving onto our main result, Theorem 2, which is an analogue of Marstrand's Theorem for a general class of dimension functions, we consider an explicit class of sets that has motivated our work and which illustrates and clarifies the need for statements such as Theorem 1 .

The motivating example. Let $\psi: \mathbb{R}^{+} \rightarrow \mathbb{R}^{+}$be a decreasing function. A point $\left(y_{1}, \ldots, y_{k}\right) \in \mathbb{R}^{k}$ is called simultaneously $\psi$-approximable if there are infinitely many $q \in \mathbb{N}$ and $\left(p_{1}, \ldots, p_{k}\right) \in \mathbb{Z}^{k}$ such that

$$
\left|y_{i}-\frac{p_{i}}{q}\right|<\psi(q) \quad 1 \leq i \leq k .
$$

The set of simultaneously $\psi$-approximable points in $\mathrm{I}^{k}:=[0,1]^{k}$ will be denoted by $W_{k}(\psi)$. For convenience, we work within the unit cube $\mathrm{I}^{k}$ rather than $\mathbb{R}^{k}$ as it makes full measure results easier to state and avoids ambiguity. This is not at all restrictive as the set of simultaneously $\psi$-approximable points is invariant under translations by integer vectors. The following statement provides a beautiful and simple criterion for the 'size' of $W_{k}(\psi)$ in terms of Hausdorff measures with respect to a dimension function $f$, see $\$ 1.2$ for the full definition of these measures.

Theorem (Khintchine-Jarník). Let $\psi: \mathbb{R}^{+} \rightarrow \mathbb{R}^{+}$be a decreasing function . Let $f$ be a dimension function such that $r^{-k} f(r)$ is monotonic. Then

$$
\mathcal{H}^{f}\left(W_{k}(\psi)\right)=\left\{\begin{array}{cccc}
0 & \text { if } & \sum_{q=1}^{\infty} q^{k} f(\psi(q))<\infty, \\
\mathcal{H}^{f}\left(\mathrm{I}^{k}\right) & \text { if } & \sum_{q=1}^{\infty} q^{k} f(\psi(q))=\infty .
\end{array}\right.
$$

This theorem unifies the fundamental results of Khintchine and Jarník in the classical theory of metric Diophantine approximation. Khintchine's Theorem (1924) corresponds to the situation in which $f(r)=r^{k}$ when $\mathcal{H}^{f}$ is equivalent to $k$-dimensional Lebesgue measure. Jarník's Theorem (1931) corresponds to the situation in which $r^{-1} f(r) \rightarrow \infty$ as $r \rightarrow 0$ and $r^{-k} f(r)$ is decreasing in which case $\mathcal{H}^{f}\left(\mathrm{I}^{k}\right)=\infty$. For background and further details see [1, 2] and references therein.

For all $\tau>0$, let $\psi_{\tau}$ be the 'approximating' function given by $\psi_{\tau}(q):=$ $\exp \left(-q^{\tau}\right)$. Then by definition, when $k=1$ the corresponding set $W_{k}\left(\psi_{\tau}\right)$ is a 
subset of the set of Liouville numbers which is well-known to be of Hausdorff dimension zero. In fact $\operatorname{dim} W_{k}\left(\psi_{\tau}\right)=0$ for all positive integers $k$. To see this, note that for any dimension function $f_{s}(r)=r^{s}(s>0)$,

$$
\sum_{q=1}^{\infty} q^{k} f_{s}\left(\psi_{\tau}(q)\right)=\sum_{q=1}^{\infty} \exp \left(-\left(s q^{\tau}-k \log q\right)\right)<\infty
$$

for all $\tau>0$ and $k \in \mathbb{R}$. Hence, it follows from the Khintchine-Jarník Theorem and the definition of Hausdorff dimension that $\operatorname{dim} W_{k}\left(\psi_{\tau}\right)=0$ for all $\tau>0$ and $k \geq 1$. The upshot of this is that by (2), for all $\theta \in[0, \pi)$

$$
\operatorname{dim}\left(\operatorname{proj}_{\theta} W_{2}\left(\psi_{\tau}\right)\right)=0
$$

and Marstrand's Theorem is not particularly informative. The problem is that the dimension functions $f_{s}$ given by $f_{s}(r)=r^{s}$ are not delicate enough to differentiate between sets of dimension zero. Instead, for $s>0$ consider the logarithmic dimension function $f_{s}$ given by $f_{s}(r)=(-\log r)^{-s}$ for $0<r<1$. Then, for $\tau>0$ and $k \geq 1$, it is easily verified that

$$
\sum_{q=1}^{\infty} q^{k} f_{s}\left(\psi_{\tau}(q)\right)=\sum_{q=1}^{\infty} q^{-(\tau s-k)}\left\{\begin{array}{ccc}
<\infty & \text { if } & s>s_{0} \\
=\infty & \text { if } & s<s_{0}
\end{array}\right.
$$

where

$$
s_{0}:=\frac{k+1}{\tau} .
$$

It then follows from the Khintchine-Jarník Theorem and the definition of logarithmic Hausdorff dimension, see \$1.2.1, that $\operatorname{dim}_{\log } W_{k}\left(\psi_{\tau}\right)=s_{0}$ for all $\tau>0$ and $k \geq 1$. In turn Theorem 1 implies the non-trivial statement that for almost all $\theta \in[0, \pi)$,

$$
\operatorname{dim}_{\log }\left(\operatorname{proj}_{\theta} W_{k}\left(\psi_{\tau}\right)\right)=s_{0}
$$

\subsection{The main result}

We first recall the definition of $f$-dimensional Hausdorff measure. Let $f: \mathbb{R}^{+} \rightarrow$ $\mathbb{R}^{+}$be a dimension or gauge function, that is a function that is increasing and continuous with $f(r) \rightarrow 0$ as $r \rightarrow 0$. Let $A$ be a non-empty subset of $\mathbb{R}^{n}$. For $\rho>0$, let

$$
\mathcal{H}_{\rho}^{f}(A):=\inf \left\{\sum_{i} f\left(\left|U_{i}\right|\right): A \subseteq \bigcup_{i} U_{i},\left|U_{i}\right| \leq \rho\right\}
$$

where $|U|$ denotes the diameter of a set $U$ and the infimum is over countable covers $\left\{U_{i}\right\}$ of $A$ by sets of diameter at most $\rho$. The Hausdorff $f$-measure of $A$ is defined by

$$
\mathcal{H}^{f}(A):=\lim _{\rho \rightarrow 0} \mathcal{H}_{\rho}^{f}(A)
$$


When $f(r)=r^{s}(s>0)$, the measure $\mathcal{H}^{f}$ is the usual $s$-dimensional Hausdorff measure $\mathcal{H}^{s}$.

We will also use centred Hausdorff measure. Here we consider covers by a countable collection of balls $\left\{B\left(x_{i}, r_{i}\right)\right\}$ of radii $r_{i} \leq \rho$ with centres in $A$. Thus, for $\rho>0$ we set

$$
\mathcal{H}_{C, \rho}^{f}(A):=\inf \left\{\sum_{i} f\left(r_{i}\right): A \subseteq \bigcup_{i} B\left(x_{i}, r_{i}\right), x_{i} \in A, r_{i} \leq \rho\right\},
$$

and define the centred Hausdorff $f$-measure of $A$ by

$$
\mathcal{H}_{C}^{f}(A):=\lim _{\rho \rightarrow 0} \mathcal{H}_{C, \rho}^{f}(A) .
$$

These two measures are equivalent, in the sense that for all $A \subseteq \mathbb{R}^{n}$

$$
\mathcal{H}_{C}^{f}(A) \leq \mathcal{H}^{f}(A) \leq m_{n} \mathcal{H}_{C}^{f}(A),
$$

where $m_{n}$ depends only on $n$. This follows easily from the definitions, noting that every set $U$ that intersects $A$ is contained in a ball with centre in $A$ and diameter $|U|$, and that every ball $B \subseteq \mathbb{R}^{n}$ of radius $r$ is contained in a finite number $m_{n}$ of balls of radius $\frac{1}{2} r$, that is diameter $r$; in particular $m_{2}=7$.

Note that $f$-Hausdorff measure only depends on $f(r)$ for $r \in\left[0, r_{0}\right]$ for arbitrarily small $r_{0}$, so changing the dimension function $f$ outside a neighbourhood of 0 does not affect the measure.

The Hausdorff dimension $\operatorname{dim} A$ of a set $A$ is defined by

$$
\operatorname{dim} A:=\inf \left\{s: \mathcal{H}^{s}(A)=0\right\}=\sup \left\{s: \mathcal{H}^{s}(A)=\infty\right\} .
$$

It follows from (5) that we get the same value for Hausdorff dimension if we replace $\mathcal{H}^{s}$ by $\mathcal{H}_{C}^{s}$ in this definition. For further discussion of Hausdorff measures and dimensions, see [6, 11, 16].

Defining Hausdorff measures for general dimension functions allows a more precise notion of dimension than just a numerical value. For example, a set $A$ may have Hausdorff dimension $s$ but with $\mathcal{H}^{s}(A)=0$. However, it may be that $0<\mathcal{H}^{f}(A)<\infty$ where, say $f(r)=r^{s} \log (1 / r)$, in which case we think of $A$ having dimension 'logarithmically smaller' than $s$. Introducing a partial order $\prec$ on the set of dimension functions by $f \prec g$ if $\lim _{r \rightarrow 0} g(r) / f(r)=0$, which implies that $\mathcal{H}^{g}(A)=0$ whenever $\mathcal{H}^{f}(A)<\infty$, allows a much finer notion of dimension, see [16]. It is also worth noting that there are sets $A \subseteq \mathbb{R}^{n}$ for which there is no dimension function $f$ such that $0<\mathcal{H}^{f}(A)<\infty$, see [4].

In order to state our main theorem we need the notion of doubling. A dimension function $f$ is said to be doubling if there exist constants $c>1$ and $r_{0}>0$ such that

$$
f(2 r) \leq c f(r) \quad \forall 0<r<r_{0} .
$$


The number $c$ is called a doubling constant. Note that if $f$ is given by $f(r)=r^{s}$ $(s>0)$ then $f(2 r)=2^{s} f(r)$ and so $c=2^{s}$ is a doubling constant for $f$.

We are now in the position to state our main result.

Theorem 2. Let $A \subseteq \mathbb{R}^{2}$ be a Borel set.

(i) Let $f$ be a dimension function. Then $\mathcal{H}^{f}\left(\operatorname{proj}_{\theta} A\right) \leq \mathcal{H}^{f}(A)$ for all $\theta \in[0, \pi)$. In particular if $\mathcal{H}^{f}(A)=0$ then $\mathcal{H}^{f}\left(\operatorname{proj}_{\theta} A\right)=0$ for all $\theta \in[0, \pi)$.

(ii) Let $f$ be a dimension function such that $\mathcal{H}^{f}(A)>0$. Suppose $g$ is a dimension function that is doubling with constant $c<2$ and such that

$$
-\int_{0}^{1} f(r) \mathrm{d}\left(\frac{1}{g(r)}\right)<\infty
$$

Then, $\mathcal{H}^{g}\left(\operatorname{proj}_{\theta} A\right)=\mathcal{H}^{g}(A)=\infty$ for almost all $\theta \in[0, \pi)$.

Several remarks are in order.

Remark 2. Part (i) of Theorem 2 is an immediate consequence of the Lipschitz condition (11) and the definition of $\mathcal{H}^{f}$, see [6, Proposition 3.1] where the case of $f(r)=r^{s}$ is given. Thus the main substance of the theorem is part (ii) when $\mathcal{H}^{f}(A)>0$.

Remark 3. Note that the conclusion of (ii) remains true if the range of integration in (17) is an interval $\left[0, r_{0}\right]$ for any $r_{0}>0$. Moreover, if $g$ is differentiable, or at least differentiable except at finitely many points, then

$$
-\int_{0}^{1} f(r) \mathrm{d}\left(\frac{1}{g(r)}\right)=\int_{0}^{1} f(r) \frac{g^{\prime}(r)}{g^{2}(r)} \mathrm{d} r .
$$

In particular, if $f$ and $g$ are dimension functions satisfying (7) then

$$
\lim _{r \rightarrow 0} \frac{f(r)}{g(r)}=0
$$

For suppose not. Then there exists $a>0$ and a sequence $r_{n} \searrow 0$ such that $f\left(r_{n}\right) / g\left(r_{n}\right) \geq a$ for all $n$. Let $r_{n}^{\prime}>r_{n}$ be the least number such that $g\left(r_{n}^{\prime}\right)=$ $2 g\left(r_{n}\right)$; such an $r_{n}^{\prime}$ exists by continuity and monotonicity of $g$ provided that the sequence is chosen taking $r_{1}$ sufficiently small. Then

$$
\int_{r_{n}}^{r_{n}^{\prime}} f(r) \frac{g^{\prime}(r)}{g^{2}(r)} \mathrm{d} r \geq \int_{r_{n}}^{r_{n}^{\prime}} \frac{f\left(r_{n}\right)}{2 g\left(r_{n}\right)} \frac{g^{\prime}(r)}{g(r)} \mathrm{d} r \geq \frac{1}{2} a \log \left[\frac{g\left(r_{n}^{\prime}\right)}{g\left(r_{n}\right)}\right]=\frac{1}{2} a \log 2 .
$$

Since $0<r_{n}<r_{n}^{\prime} \rightarrow 0$, the integrals in (8) and (7) cannot be finite. 
When contemplating an extension of Marstrand's theorem to general dimension functions, it is not unreasonable to suspect a statement along the lines of Theorem 2 with condition (77) replaced by (99). The latter condition is natural and it initially appears to avoid known examples (see for instance [11, 9.2 Example]) of $s$-sets $A \subseteq \mathbb{R}^{2}$ with $0<s \leq 1$ for which $\mathcal{H}^{s}\left(\operatorname{proj}_{\theta} A\right)=0$ for all $\theta \in[0, \pi)$. As we shall see in the appendix the construction of these sets can be adapted to show that we can not in general replace condition (7) by (9) in Theorem 2. The following statement is easily deduced from the theorem proved in the appendix for codoubling dimension function. A dimension function $f$ is said to be codoubling if there exist constants $c>1$ and $r_{0}>0$ such that

$$
f(2 r) \geq c f(r) \quad \forall 0<r<r_{0} .
$$

Theorem $\mathbf{A}^{\prime}$. Let $f, g$ be dimension functions such that $f$ is doubling with constant $c \leq 2$ and codoubling, and such that

$$
g(r) \leq M f\left(r \log \left(r^{-1}\right)\right) \quad \forall 0<r<r_{0}
$$

where $r_{0}>0$ and $M>0$ are constants. Then there exists a set $A \subseteq \mathbb{R}^{2}$ with $0<\mathcal{H}^{f}(A)<\infty$ but $\mathcal{H}^{g}\left(\operatorname{proj}_{\theta} A\right)=0$ for all $0 \leq \theta<\pi$.

It is easily seen that Theorem $\mathrm{A}^{\prime}$ is not a converse to Theorem 2. Namely, fix some $\delta \in(0,1)$ and for $s>0$ consider dimension functions $g_{s}$ given by

$$
g_{s}(r)=r^{\delta} \log ^{s}\left(r^{-1}\right) .
$$

Clearly, $g_{s}$ is doubling with constant $c<2$. Also, let $f$ be given by $f(r)=r^{\delta}$. Clearly, $f$ is doubling with constant $c \leq 2$ and codoubling. Then for any $s \leq \delta$, condition (11) is satisfied and Theorem $\mathrm{A}^{\prime}$ implies that there exists a set $A_{s}$ with positive $\delta$-dimensional Hausdorff measure such that $\mathcal{H}^{g_{s}}\left(\operatorname{proj}_{\theta} A_{s}\right)=0$ for all $0 \leq \theta<\pi$. On the other hand, for any $s \geq 1$, condition (77) is satisfied and Theorem 2 implies that there does not exist such a set $A_{s}$. But for $s \in(\delta, 1)$, we do not know whether such a set exists. Nevertheless, Theorem $A^{\prime}$ shows that we can not in general replace condition (7) by (91) and thus there is a gap of uncertainty associated with Theorem 2 where we do not know what happens (for further discussion see $\$ 4$ ). This gap of uncertainty will be explicitly highlighted in $\$ 1.2 .2$ when we return to our motivating example. The fact that condition (7) shows up is very much a consequence of the approach taken to prove the theorem. Concerning this the reader is especially directed towards Remark 7 at the end of 22.2 in the proof.

Not surprisingly, condition (11) implies that the integral convergence condition (9) is violated - see the appendix for the details; namely Remark 12 . 
Remark 4. It is easily verified that if $f$ and $g$ are dimension functions satisfying (9) and $\mathcal{H}^{f}(A)>0$ then $\mathcal{H}^{g}(A)=\infty$. Thus, the main substance of part (ii) of Theorem 22 is the statement that $\mathcal{H}^{g}\left(\operatorname{proj}_{\theta} A\right) \geq \mathcal{H}^{g}(A)$ for almost all $\theta \in[0, \pi)$. For further relations between measures with respect to different gauge functions, see [16, Section 4].

Remark 5. Regarding the dimension function $g$, the condition that $c<2$ on the doubling constant is necessary. To see this, we derive the dimension aspect of Marstrand's Theorem from our result. With this in mind, assume without loss of generality that $\operatorname{dim} A>0$ and let $s_{1}, s_{2}$ be arbitrary real numbers satisfying $0<s_{1}<s_{2}<\operatorname{dim} A$. Now let $g$ and $f$ be dimension functions given by $g(r):=$ $r^{s_{1}}$ and $f(r):=r^{s_{2}}$. It follows from the definition of Hausdorff dimension that $\mathcal{H}^{s_{1}}(A)=\mathcal{H}^{s_{2}}(A)=\infty$. Also it is easily checked that condition (77) is satisfied and thus, modulo the condition on the doubling constant, part (ii) of Theorem 2 implies that $\mathcal{H}^{s_{1}}\left(\operatorname{proj}_{\theta} A\right)=\infty$ for almost all $\theta \in[0, \pi)$. In turn, it follows (from the definition of Hausdorff dimension) that

$$
\operatorname{dim} \operatorname{proj}_{\theta} A \geq s_{1}
$$

for almost all $\theta \in[0, \pi)$. The application of Theorem 2 is legitimate as long as the doubling constant $c=2^{s_{1}}$ associated with $g$ satisfies $s_{1}<1$. Now with reference to (12) this restriction on $s_{1}$ makes perfect sense since $\operatorname{dim} \operatorname{proj}_{\theta} A \leq 1$ regardless of the size of $A$. By continuity, we can replace $s_{1}$ in (12) by $\operatorname{dim} A$. The complementary upper bound can easily be deduced via part (i) of Theorem 2 but inequality (2) gives it directly.

Remark 6. Even if $\mathcal{H}^{f}(A)=\infty$, the conclusion of part (ii) of Theorem 2 is not in general valid for the dimension function $f$. Indeed, if $f$ is given by $f(r):=r$ so that $\mathcal{H}^{f}$ is simply 1-dimension Lebesgue measure, it is known [6, Section 6.4] that there are sets $A$ for which $\mathcal{H}^{f}(A)>0$ but $\mathcal{H}^{f}\left(\operatorname{proj}_{\theta} A\right)=0$ for almost all $\theta \in[0, \pi)$.

As alluded to in Remark 1, in $\$ 3$ we will investigate the size of the set of exceptional angles $\theta$ for which the conclusion of part (ii) of Theorem 2 fails. In short, by replacing the integral convergence condition (7) by a suitable rate of convergence condition we are able to conclude that the exceptional set of $\theta \in[0, \pi)$ for which $\mathcal{H}^{g}\left(\operatorname{proj}_{\theta} A\right)<\infty$ is of $\mathcal{H}^{f}$-measure 0 , see Theorem 9 for the precise statement.

\subsubsection{The logarithmic dimension result}

In terms of dimension theory, when we are confronted with sets of Hausdorff dimension 0 it is natural to change the usual ' $r{ }^{s}$-scale' in the definition of Hausdorff dimension to a logarithmic scale. For $s>0$, let $f_{s}$ be the dimension function 
given by $f_{s}(r):=\left(-\log ^{*} r\right)^{-s}$, where

$$
\log ^{*} r:= \begin{cases}\log r & \text { for } r \in\left(0, \frac{1}{2}\right) \\ \log \frac{1}{2} & \text { for } r \geq \frac{1}{2}\end{cases}
$$

[The form of $\log ^{*} r$ for $r \geq \frac{1}{2}$ is to ensure that it is defined for $r \geq 1$; as remarked earlier, the particular form for $r \geq \frac{1}{2}$ is of no consequence.] The logarithmic Hausdorff dimension $\operatorname{dim}_{\log } A$ of a set $A$ is given by

$$
\operatorname{dim}_{\log } A:=\inf \left\{s: \mathcal{H}^{f_{s}}(A)=0\right\}=\sup \left\{s: \mathcal{H}^{f_{s}}(A)=\infty\right\} .
$$

It is easily verified that if $\operatorname{dim} A>0$ then $\operatorname{dim}_{\log } A=\infty$, precisely as one would expect.

Armed with Theorem 2 it is straightforward to prove Theorem 1 .

Proof of Theorem 1. Part (i) is immediate from Theorem 2(i) and (13).

For part (ii), without loss of generality, assume that $\operatorname{dim}_{\log } A>0$ and let $s_{1}, s_{2}$ be real numbers satisfying $0<s_{1}<s_{2}<\operatorname{dim}_{\log } A$. Let $g$ and $f$ be dimension functions given by $g(r):=\left(-\log ^{*} r\right)^{-s_{1}}$ and $f(r):=\left(-\log ^{*} r\right)^{-s_{2}}$. It follows from (13) that $\mathcal{H}^{g}(A)=\mathcal{H}^{f}(A)=\infty$. It is easily verified that condition (17) is satisfied and that $g$ is doubling with constant $c<2$ for $r \in\left(0, r_{0}\right)$ for some $r_{0}$. Thus, Theorem 2(ii) implies that $\mathcal{H}^{g}\left(\operatorname{proj}_{\theta} A\right)=\infty$ for almost all $\theta \in[0, \pi)$. In turn, it follows from (13) that for almost all $\theta \in[0, \pi), \operatorname{dim}_{\log } \operatorname{proj}_{\theta} A \geq s_{1}$ and thus $\operatorname{dim}_{\log } \operatorname{proj}_{\theta} A \geq \operatorname{dim}_{\log } A$.

\subsubsection{Explicitly exposing the gap of uncertainty}

With reference to our motivating example, for all $\tau>0$ let $\psi_{\tau}$ be the 'approximating' function given by

$$
\psi_{\tau}(q):=q^{-\tau}(\log q)^{-\tau} \quad(q \geq 1)
$$

It follows, via the Khintchine-Jarník Theorem and the definition of Hausdorff dimension, that for all $\tau \geq(1+k) / k$

$$
\delta=\delta(\tau):=\operatorname{dim} W_{k}\left(\psi_{\tau}\right)=\frac{k+1}{\tau} .
$$

In fact, the Khintchine-Jarník Theorem implies a much finer conclusion. Fix $\tau>(1+k) / k$ and consider the family of dimension functions $\left(f_{\delta, s}\right)_{s>0}$ given by

$$
f_{\delta, s}(r):=r^{\delta}\left(-\frac{1}{\tau} \log ^{*} r\right)^{s}
$$


It is easily verified that

$$
\sum_{q=2}^{\infty} q^{k} f_{\delta, s}\left(\psi_{\tau}(q)\right) \asymp \sum_{q=2}^{\infty} \frac{1}{q(\log q)^{1+k-s}},
$$

in the sense that the series either both converge or diverge, and so the KhintchineJarník Theorem implies that

$$
\mathcal{H}^{f_{\delta, s}}\left(W_{k}\left(\psi_{\tau}\right)\right)= \begin{cases}0 & \text { if } \quad s<k \\ \infty & \text { if } \quad s \geq k\end{cases}
$$

Loosely speaking, the set $W_{k}\left(\psi_{\tau}\right)$ has " $\delta(\tau)$-logarithmic dimension" equal to $k$.

Now let $k=2$ and with reference to Theorem 2, put $f=f_{\delta, 2}$ and $g=f_{\delta, s}$. Suppose that $\tau>3$ so that $\delta(\tau)<1$. This ensures that $g$ is doubling with constant $c<2$. Theorem 2 then implies that for almost all $\theta \in[0, \pi)$

$$
\mathcal{H}^{f_{\delta, s}}\left(\operatorname{proj}_{\theta} W_{2}\left(\psi_{\tau}\right)\right)=\left\{\begin{array}{ccc}
0 & \text { if } & s<2, \\
\infty & \text { if } & s>3
\end{array}\right.
$$

Of course, part (i) of Theorem 2 implies that the zero measure statement associated with $s<2$ is true for all $\theta \in[0, \pi)$. Regarding the application of part (ii), we need that $s>3$ in order to satisfy the integral convergence condition (17). Thus the latter gives rise to a gap of uncertainty; namely $s \in(2,3)$ in the specific example under consideration. We suspect that the infinity measure statement for $s>3$ is actually true for $s>2$.

Problem: Show that $\mathcal{H}^{f_{\delta, s}}\left(\operatorname{proj}_{\theta} W_{2}\left(\psi_{\tau}\right)\right)=\infty$ if $s>2$.

The fact of the matter is that it is highly unlikely that any set $W_{2}(\psi)$ of simultaneously $\psi$-approximable points will have the necessary 'dense rotational' structure that underpins the construction of the sets associated with Theorem $\mathrm{A}^{\prime}$.

\section{Proof of main result}

Our proof of Theorem 2 will follow Kaufman's potential theoretic proof [9] of Mastrand's Theorem. We adapt the proof that he gave for the specific functions $f(r)=r^{s}(s>0)$ to general dimension functions.

\subsection{Preliminaries: doubling revisited and Frostman}

We start by stating an equivalent form of the doubling condition (6). 
Lemma 3. Let $f$ be a dimension function. Then $f$ is doubling if and only if there exist constants $s>0, \kappa>0$ and $r_{1}>0$ such that

$$
f(r \lambda) \geq \kappa \lambda^{s} f(r) \quad \forall 0<\lambda<1 \text { and } 0<r<r_{1} .
$$

Moreover, if $f$ has a doubling constant $c>1$ then (14) holds with $\kappa=c^{-1}$ and $s=\log _{2} c$.

This equivalence is essentially 'folklore' and the exponent $s$ appearing in (14) is referred to as the doubling exponent of $f$. Nevertheless, for the sake of completeness we include the short proof.

Proof. Suppose $f$ has a doubling constant $c>1$. For each positive integer $n$, applying (6) $n$ times gives that

$$
f(r) \leq c^{n} f\left(2^{-n} r\right) \quad \forall r<2^{n} r_{0} .
$$

Put $s:=\log _{2} c>0$. For each $0<\lambda<1$, let $m \geq 0$ be the unique integer such that $2^{m} \leq \lambda^{-1}<2^{m+1}$. Then

$$
f(\lambda r) \geq f\left(2^{-(m+1)} r\right) \geq c^{-(m+1)} f(r) \geq c^{-1} \lambda^{s} f(r) .
$$

For the converse implication simply put $\lambda=\frac{1}{2}$ in (14).

The following statement is a generalisation of Frostman's fundamental lemma to arbitrary dimension functions $f$. Throughout, given a Borel set $A \subseteq \mathbb{R}^{2}$ we denote by $\mathcal{M}_{1}(A)$ the set of Radon probability measures $\mu$ with compact support in $A$.

Theorem 4 (Frostman's Lemma). Let $A \subseteq \mathbb{R}^{2}$ be a Borel set and $f$ be a dimension function. Then $\mathcal{H}^{f}(A)>0$ if and only if there exist a measure $\mu \in \mathcal{M}_{1}(A)$ and a constant $c_{1}>0$ such that

$$
\mu(B(x, r)) \leq c_{1} f(r) \quad \forall x \in \mathbb{R}^{2} \text { and } r>0 .
$$

Proof. Two very different proofs for the case where $f(r)=r^{s}(s>0)$ are given in [11, Theorem 8.8], where is explicitly pointed out that both proofs are valid for general dimension functions. Alternatively, for the harder of the implications, namely that suitable measures exist, the result in Rogers [16, Theorem 57], that for general dimension functions there exists a compact subset $A^{\prime}$ of $A$ with $0<$ $\mathcal{H}^{f}\left(A^{\prime}\right)<\infty$, followed by a density argument akin to [6, Proposition 4.11], also gives the conclusion. 


\section{$2.2 \quad$ Energies and capacities}

We first generalise the standard notions of $s$-energy and $s$-capacity of a measure, see for example [6, Section 4.3] and [11, Chapter 8]. As usual, let $f$ be a dimension function.

Definition 5. The $f$-energy of $\mu \in \mathcal{M}_{1}(A)$ is defined as

$$
I_{f}(\mu):=\iint \frac{\mathrm{d} \mu(x) \mathrm{d} \mu(y)}{f(|x-y|)} .
$$

Alternatively, we could have defined the $f$-energy via the $f$-potential at a point $x \in \mathbb{R}^{2}$, that is

$$
\phi_{f}(x):=\int \frac{\mathrm{d} \mu(y)}{f(|x-y|)} \quad \text { and so } \quad I_{f}(\mu)=\int \phi_{f}(x) \mathrm{d} \mu(x) .
$$

Definition 6. The $f$-capacity of a Borel set $A \subseteq \mathbb{R}^{2}$ is defined as

$$
C_{f}(A):=\sup \left\{\frac{1}{I_{f}(\mu)}: \mu \in \mathcal{M}_{1}(A)\right\}
$$

with the interpretation that $C_{f}(\emptyset)=0$.

Naturally, when $f$ is given by $f(r)=r^{s}(s>0)$ we recover the familiar notions of $s$-energy and $s$-capacity.

We now establish the connection between the Hausdorff measure $\mathcal{H}^{f}(A)$ and the capacity $C_{f}(A)$ of a set $A$ with respect to a general dimension function $f$. These results stated below have a long history: apart from notational differences they appear as Theorems 1 and 2 in [17, though versions for the dimension functions of the form $f(r)=r^{s}$ date back to the 1930s. The paper [17] discusses the historical development to increasingly general dimension functions and includes further references. Proofs for dimension functions $f(r)=r^{s}$ may be found in several more recent accounts of fractal geometry, for example [5, 11]. Even for general dimension functions the proofs are relatively short, so for the sake of clarity, consistency of notation and completeness we include the proofs.

Proposition 7. Let $A \subseteq \mathbb{R}^{2}$ be a Borel set and $f$ be a dimension function. If $\mathcal{H}^{f}(A)<\infty$ then $C_{f}(A)=0$.

Proof. Assume $C_{f}(A)>0$. By definition, the set $A$ supports a Radon probability measure $\mu$ such that $I_{f}(\mu)<\infty$. Thus

$$
\int \frac{\mathrm{d} \mu(y)}{f(|x-y|)}<\infty \quad \text { for } \mu \text {-almost all } x \in A .
$$


For such $x \in A$,

$$
\lim _{r \rightarrow 0} \int_{B(x, r)} \frac{\mathrm{d} \mu(y)}{f(|x-y|)}=0 .
$$

By Egorov's theorem, for all $\varepsilon>0$ there exist $\delta>0$ and a Borel set $K \subseteq A$ such that $\mu(K)>\frac{1}{2}$ and

$$
\begin{aligned}
\mu(B(x, r)) & \leq f(r) \int_{B(x, r)} \frac{\mathrm{d} \mu(y)}{f(|x-y|)} \\
& \leq \varepsilon f(r) \quad \text { for all } x \in K \text { and } 0<r \leq \delta .
\end{aligned}
$$
that

Now let $\left(B\left(x_{i}, r_{i}\right)\right)_{i=1}^{\infty}$ be a cover of $K$ by balls with $x_{i} \in K$ and $r_{i} \leq \delta$ such

$$
\sum_{i=1}^{\infty} f\left(r_{i}\right)<\mathcal{H}_{C}^{f}(K)+1
$$

Then

$$
\frac{1}{2}<\mu(K) \leq \sum_{i=1}^{\infty} \mu\left(B\left(x_{i}, r_{i}\right)\right) \leq \varepsilon \sum_{i=1}^{\infty} f\left(r_{i}\right) \leq \varepsilon\left(\mathcal{H}_{C}^{f}(A)+1\right),
$$

where $\mathcal{H}_{C}^{f}$ is centred Hausdorff measure. Since $\varepsilon>0$ can be made arbitrarily small, we conclude that $\mathcal{H}^{f}(A)=\mathcal{H}_{C}^{f}(A)=\infty$, using (5). This contradicts our hypothesis that $\mathcal{H}^{f}(A)$ is finite.

Proposition 8. Let $A \subseteq \mathbb{R}^{2}$ be a Borel set and let $f$ and $g$ be dimension functions satisfying the integral convergence condition (7). If $\mathcal{H}^{f}(A)>0$ then $C_{g}(A)>0$.

Proof. By Frostman's lemma, Theorem [4 the Borel set $A$ supports a Radon probability measure $\mu$ such that

$$
\mu(B(x, r)) \leq c_{1} f(r) \quad \forall x \in \mathbb{R}^{2} \text { and } r>0
$$

for some constant $c_{1}>0$. Fix $x \in \mathbb{R}^{2}$ and let

$$
m(r):=\mu(B(x, r)) .
$$


Using (15) and that $\mu\left(\mathbb{R}^{2}\right)=\mu(K)=1$ and integrating by parts,

$$
\begin{aligned}
\int \frac{\mathrm{d} \mu(y)}{g(|x-y|)} & =\int_{|x-y| \leq 1} \frac{\mathrm{d} \mu(y)}{g(|x-y|)}+\int_{|x-y|>1} \frac{\mathrm{d} \mu(y)}{g(|x-y|)} \\
& \leq \int_{0}^{1} \frac{1}{g(r)} \mathrm{d} m(r)+\frac{\mu\left(\mathbb{R}^{2}\right)}{g(1)} \\
& =\left[\frac{m(r)}{g(r)}\right]_{0}^{1}-\int_{0}^{1} m(r) \mathrm{d}\left(\frac{1}{g(r)}\right)+\frac{\mu\left(\mathbb{R}^{2}\right)}{g(1)} \\
& \leq \frac{m(1)}{g(1)}-\lim _{r \rightarrow 0^{+}} \frac{m(r)}{g(r)}-\int_{0}^{1} f(r) \mathrm{d}\left(\frac{1}{g(r)}\right)+\frac{\mu\left(\mathbb{R}^{2}\right)}{g(1)} \\
& <\infty,
\end{aligned}
$$

noting that $m(r) / g(r) \leq c_{1} f(r) / g(r) \rightarrow 0$ by (9). This bound is uniform for all $x \in \mathbb{R}$ and so

$$
I_{g}(\mu)=\iint \frac{\mathrm{d} \mu(x) \mathrm{d} \mu(y)}{g(|x-y|)}<\infty
$$

giving $C_{g}(A)>0$ by Definition 6 .

Remark 7. Fix $0<\delta<1$ and consider the family of dimension functions $\left(f_{\delta, s}\right)_{s>0}$ given by

$$
f_{\delta, s}(r):=r^{\delta}\left(-\log ^{*} r\right)^{s},
$$

to within constants the same as those considered in $\$ 1.2 .2$. Let $A \subseteq \mathbb{R}^{2}$ be a Borel set and $\alpha>0$. Then, by Propositions 7 and 8 ,

(i) if $s \leq \alpha$ and $\mathcal{H}^{f_{\delta, \alpha}}(A)<\infty$ then $C_{f_{\delta, s}}(A)=0$,

(ii) if $s>\alpha+1$ and $\mathcal{H}^{f_{\delta, \alpha}}(A)>0$ then $C_{f_{\delta, s}}(A)>0$.

The upshot is that if $\alpha<s \leq \alpha+1$, condition (7) is not satisfied and the propositions provide no information. The main aim of the paper [17] is to expose this gap of uncertainty. So for example, by [17, Theorem 3], if $f$ and $g$ are dimension functions not satisfying condition (7), then there exist Borel sets $A$ with $0<\mathcal{H}^{f}(A)<\infty$ but $C_{g}(A)=0$. 


\subsection{Proof of Theorem 2}

(i) As pointed out in Remark 2, this is a trivial consequence of the definition of the Hausdorff measures that projection is a Lipschitz mapping.

(ii) From Remark 4, $\mathcal{H}^{g}(A)=\infty$. Thus it suffices to show that $\mathcal{H}^{g}\left(\operatorname{proj}_{\theta} A\right)=\infty$ for almost all $\theta \in[0, \pi)$.

Since $\mathcal{H}^{f}(A)>0$, it follows via Proposition 8 and the definition of capacity, that $A$ supports a Radon probability measure $\mu$ such that $I_{g}(\mu)<\infty$. For each $\theta \in[0, \pi)$, projecting $\mu$ onto the line $L_{\theta}$ gives a measure $\mu_{\theta}$ supported on $\operatorname{proj}_{\theta} A$ defined by the requirement that $\mu_{\theta}(K)=\mu\left(\operatorname{proj}_{\theta}^{-1}(K)\right)$ for each Borel set $K \subseteq L_{\theta}$. For each $x \in \mathbb{R}^{2}$, let $\phi(x)$ denote the angle that $x$ (viewed as a vector) forms with the horizontal axis. Then, by Lemma 3 and using the fact that $g$ is doubling with constant $c<2$, it follows that

$$
\begin{aligned}
\int_{0}^{\pi} I_{g}\left(\mu_{\theta}\right) \mathrm{d} \theta & =\int_{0}^{\pi} \iint \frac{\mathrm{d} \mu_{\theta}(x) \mathrm{d} \mu_{\theta}(y)}{g(|x-y|)} \mathrm{d} \theta \\
& =\int_{0}^{\pi} \iint \frac{\mathrm{d} \mu(x) \mathrm{d} \mu(y)}{g\left(\left|\operatorname{proj}_{\theta} x-\operatorname{proj}_{\theta} y\right|\right)} \mathrm{d} \theta \\
& \leq \iint\left(\int_{0}^{\pi} \frac{c}{g(|x-y|)|\cos (\phi(x-y)-\theta)| s} \mathrm{~d} \theta\right) \mathrm{d} \mu(x) \mathrm{d} \mu(y) \\
& \left.\leq c_{1} \iint \frac{\mathrm{d} \mu(x) \mathrm{d} \mu(y)}{g(|x-y|)} \quad \text { (because } s=\log _{2} c<1\right) \\
& =c_{1} I_{g}(\mu)<\infty .
\end{aligned}
$$

This implies that $I_{g}\left(\mu_{\theta}\right)<\infty$ for almost all $\theta \in[0, \pi)$. From the definition of capacity, $C_{g}\left(\operatorname{proj}_{\theta} A\right)>0$ for such $\theta$, so by Proposition $7, \mathcal{H}^{g}\left(\operatorname{proj}_{\theta} A\right)=\infty$ for almost all $\theta \in[0, \pi)$.

\section{$3 \quad$ Exceptional projections}

Marstrand's Theorem trivially implies that the set of exceptional angles

$$
E(A):=\left\{\theta \in[0, \pi): \operatorname{dim} \operatorname{proj}_{\theta} A<\operatorname{dim} A\right\},
$$

is a set of (one-dimensional) Lebesgue measure zero. Kaufman also showed [9] that

$$
\operatorname{dim} E(A) \leq \min \{1, \operatorname{dim} A\}
$$


(see also Remark 8 below). Clearly, when $\operatorname{dim} A<1$, this bound on the size of the set of exceptional angles is significantly stronger than the measure zero statement of Marstrand's Theorem. It is natural to attempt to extend Theorem 2 in a similar fashion. With this in mind, let $E_{g}(A)$ denote the exceptional set of $\theta \in[0, \pi)$ for which the conclusion of part (ii) of Theorem 2 fails; that is

$$
E_{g}(A):=\left\{\theta \in[0, \pi): \mathcal{H}^{g}\left(\operatorname{proj}_{\theta} A\right)<\infty\right\}
$$

By replacing the integral convergence condition (7) by a rate of convergence condition we are able to establish the following strengthening of Theorem 2, It is easily verified that condition (18) below implies condition (7) of Theorem 2.

Theorem 9. Let $A \subseteq \mathbb{R}^{2}$ be a Borel set. Let $f$ be a dimension function such that $\mathcal{H}^{f}(A)>0$ and let $g$ be a dimension function that is doubling. Suppose that there exist constants $t_{0}$ and $c_{2}>0$ such that

$$
-\int_{0}^{1} f(r) \mathrm{d}\left(\frac{1}{g(t r)}\right)<c_{2} \frac{1}{g(t)} \quad \text { for all } 0<t<t_{0} .
$$

Then, $\mathcal{H}^{f}\left(E_{g}(A)\right)=0$.

Proof. In view of Proposition 7,

$$
E_{g}(A) \subseteq E_{*}:=\left\{\theta \in[0, \pi): C_{g}\left(\operatorname{proj}_{\theta} A\right)=0\right\} .
$$

Thus, it suffices to show that $\mathcal{H}^{f}\left(E_{*}\right)=0$. Suppose this is not the case. Then $\mathcal{H}^{f}\left(E_{*}\right)>0$ and by Theorem 4 the set $E_{*}$ supports a probability measure $\nu \in$ $\mathcal{M}_{1}\left(E_{*}\right)$ such that

$$
\nu(B(x, r)) \leq c_{1} f(r) \text { for all } x \in \mathbb{R}^{2} \text { and } r>0
$$

where $c_{1}>0$ is an absolute constant. On the other hand, since $\mathcal{H}^{f}(A)>0$ and condition (18) implies condition (7), it follows via Proposition 8 and the definition of capacity, that $A$ supports a probability measure $\mu \in \mathcal{M}_{1}(A)$ such that

$$
I_{g}(\mu)<\infty
$$

For each $\theta \in[0, \pi)$, let $\mu_{\theta}$ be the projection of $\mu$ onto the line $L_{\theta}$ supported on $\operatorname{proj}_{\theta} A$ such that $\mu_{\theta}(K)=\mu\left(\operatorname{proj}_{\theta}^{-1}(K)\right)$ for each Borel set $K \subseteq L_{\theta}$ - as in the proof of Theorem 2. Let us assume for the moment that

$$
\int_{E_{*}} I_{g}\left(\mu_{\theta}\right) \mathrm{d} \nu(\theta)<\infty
$$

This implies that $I_{g}\left(\mu_{\theta}\right)<\infty$ for $\nu$-almost all $\theta \in E_{*}$. By the definition of capacity, $C_{g}\left(\operatorname{proj}_{\theta} A\right)>0$ for such $\theta$, contradicting that $C_{g}\left(\operatorname{proj}_{\theta} A\right)=0$ if $\theta \in E_{*}$. This completes the proof of the theorem modulo establishing (20). 
To establish (20), we first observe that for all $x \in \mathbb{R}^{2} \backslash\{0\}$ and $d>0$, the set

$$
\left\{\theta \in[0, \pi):\left|\operatorname{proj}_{\theta} x\right| \leq d\right\}
$$

is a union of at most two intervals each of diameter at most $\pi d /|x|$. The upshot is that

$$
\nu\left(\left\{\theta \in[0, \pi):\left|\operatorname{proj}_{\theta} x\right| \leq d\right\}\right) \leq 2 c_{1} f\left(\pi \frac{d}{|x|}\right) .
$$

This, together with the fact that $g$ is doubling, implies that

$$
\begin{aligned}
& \int_{E_{*}} \frac{1}{g\left(\left|\operatorname{proj}_{\theta} x\right|\right)} \mathrm{d} \nu(\theta)=\int_{0}^{\infty} \nu\left(\left\{\theta: \frac{1}{g\left(\left|\operatorname{proj}_{\theta} x\right|\right)} \geq r\right\}\right) \mathrm{d} r \\
& =\int_{0}^{1 / g(|x|)} \nu\left(\left\{\theta: \frac{1}{g\left(\left|\operatorname{proj}_{\theta} x\right|\right)} \geq r\right\}\right) \mathrm{d} r \\
& +\quad \int_{1 / g(|x|)}^{\infty} \nu\left(\left\{\theta: \frac{1}{g\left(\left|\operatorname{proj}_{\theta} x\right|\right)} \geq r\right\}\right) \mathrm{d} r \\
& \leq \frac{1}{g(|x|)}+\int_{1 / g(|x|)}^{\infty} 2 c_{1} f\left(\frac{\pi}{|x|} g^{-1}\left(\frac{1}{r}\right)\right) \mathrm{d} r \\
& \leq \frac{1}{g(|x|)}-2 c_{1} \int_{0}^{\pi} f(u) \mathrm{d}\left(\frac{1}{g(|x| u / \pi)}\right) \\
& =\frac{1}{g(|x|)}+2 c_{1} \int_{0}^{1} f(u) \mathrm{d}\left(\frac{-1}{g(|x| u / \pi)}\right) \\
& +2 c_{1} \int_{1}^{\pi} f(u) \mathrm{d}\left(\frac{-1}{g(|x| u / \pi)}\right) \\
& \stackrel{\sqrt[118]{\leq}}{\leq} \frac{1}{g(|x|)}+2 c_{1} c_{2} \frac{1}{g(|x| / \pi)} \\
& +2 c_{1} f(\pi)\left(\frac{1}{g(|x| / \pi)}-\frac{1}{g(|x|)}\right) \\
& \leq c_{3} \frac{1}{g(|x|)}
\end{aligned}
$$


for some $c_{3}$ and for all $x \neq 0$ with $|x|<t_{0}$. Hence, using Fubini's theorem,

$$
\begin{aligned}
\int_{E_{*}} I_{g}\left(\mu_{\theta}\right) \mathrm{d} \nu(\theta) & =\int_{E_{*}} \iint \frac{\mathrm{d} \mu_{\theta}(x) \mathrm{d} \mu_{\theta}(y)}{g(|x-y|)} \mathrm{d} \nu(\theta) \\
& =\int E_{E_{*}} \iint \frac{\mathrm{d} \mu(x) \mathrm{d} \mu(y)}{g\left(\left|\operatorname{proj}_{\theta} x-\operatorname{proj}_{\theta} y\right|\right)} \mathrm{d} \nu(\theta) \\
& =\iiint_{E_{*}} \frac{\mathrm{d} \nu(\theta)}{g\left(\left|\operatorname{proj}_{\theta}(x-y)\right|\right)} \mathrm{d} \mu(x) \mathrm{d} \mu(y) \\
& \leq c_{3} \iint \frac{\mathrm{d} \mu(x) \mathrm{d} \mu(y)}{g(|x-y|)}<\infty
\end{aligned}
$$

by (19). This establishes (20) and completes the proof.

Remark 8. The above proof of Theorem 9 is based on the proof of the dimension inequality (16) presented in [12, Theorem 5.1]. Note that it is easy to deduce (16) from Theorem 9. Indeed, to see that this is the case, without loss of generality assume that $0<\operatorname{dim} A<1$ and let $s_{1}, s_{2}$ be real numbers satisfying $0<s_{1}<$ $s_{2}<\operatorname{dim} A$. Let

$$
E\left(A, s_{1}\right):=\left\{\theta \in[0, \pi): \operatorname{dim}_{\operatorname{proj}} A<s_{1}\right\} .
$$

Let $g$ and $f$ be dimension functions given by $g(r):=r^{s_{1}}$ and $f(r):=r^{s_{2}}$. It follows that $\mathcal{H}^{s_{2}}(A)=\infty$ and that $\mathcal{H}^{s_{1}}\left(\operatorname{proj}_{\theta} A\right)=0$ for all $\theta \in E\left(A, s_{1}\right)$. Thus

$$
E\left(A, s_{1}\right) \subseteq E_{g}(A)
$$

with $E_{g}(A)$ as in (17). Clearly, the function $g$ is doubling and it is easily checked that $f$ and $g$ satisfy condition (18). Theorem 9 implies that $\mathcal{H}^{s_{2}}\left(E_{g}(A)\right)=0$ and so $\operatorname{dim}\left(E\left(A, s_{1}\right)\right) \leq s_{2}$, and (16) follows on taking $s_{1}, s_{2}$ arbitrarily close to $\operatorname{dim} A$.

Remark 9. The above proof of Theorem 9 is based on the proof of the special case (16) presented in [12, Theorem 5.1].

Armed with Theorem 9 it is straightforward to prove (3) which we formally state as a corollary.

Corollary 10. Let $A \subseteq \mathbb{R}^{2}$ be a Borel set. Then, $\operatorname{dim}_{\log } E_{\log }(A) \leq \operatorname{dim}_{\log } A$ where

$$
E_{\log }(A):=\left\{\theta \in[0, \pi): \operatorname{dim}_{\log } \operatorname{proj}_{\theta}(A)<\operatorname{dim}_{\log } A\right\}
$$


Proof. Without loss of generality, assume that $0<\operatorname{dim}_{\log } A<\infty$ and let $s_{1}, s_{2}$ be real numbers satisfying $0<s_{1}<s_{2}<\operatorname{dim}_{\log } A$. Let

$$
E_{\log }\left(A, s_{1}\right):=\left\{\theta \in[0, \pi): \operatorname{dim}_{\log } \operatorname{proj}_{\theta}(A)<s_{1}\right\} .
$$

As in the proof of Theorem 1, let $g$ and $f$ be the dimension functions $g(r):=$ $\left(-\log ^{*} r\right)^{-s_{1}}$ and $f(r):=\left(-\log ^{*} r\right)^{-s_{2}}$. Then $\mathcal{H}^{f}(A)=\infty$ and $\mathcal{H}^{g}\left(\operatorname{proj}_{\theta}(A)\right)=0$ for all $\theta \in E_{\log }\left(A, s_{1}\right)$ so $E_{\log }\left(A, s_{1}\right) \subseteq E_{g}(A)$. Clearly $g$ is doubling. Assume for the moment that $f$ and $g$ satisfy condition (18). Then Theorem 9 implies that $\mathcal{H}^{f}\left(E_{g}(A)\right)=0$ so from the definition of logarithmic Hausdorff dimension (13),

$$
\operatorname{dim}_{\log }\left(E_{\log }\left(A, s_{1}\right)\right) \leq s_{2} .
$$

The conclusion now follows on taking $s_{1}, s_{2}$ arbitrarily close to $\operatorname{dim}_{\log } A$.

It remains to verify (18). For all sufficiently small $t>0$,

$$
\begin{aligned}
-\int_{0}^{1} f(r) \mathrm{d}\left(\frac{1}{g(t r)}\right) & =\left[\frac{f(r)}{g(t r)}\right]_{1}^{0}+\int_{0}^{1} \frac{\mathrm{d} f(r)}{g(t r)} \\
& =-\frac{f(1)}{g(t)}+\int_{0}^{t} \frac{\mathrm{d} f(r)}{g(t r)}+\int_{t}^{1} \frac{\mathrm{d} f(r)}{g(t r)}
\end{aligned}
$$

For the first integral, $r \leq t$ implies that

$$
\frac{1}{g(t r)} \leq 2^{s_{1}}(-\log r)^{s_{1}}=2^{s_{1}} \frac{1}{g(r)}
$$

and hence it follows that

$$
\begin{aligned}
\int_{0}^{t} \frac{\mathrm{d} f(r)}{g(t r)} & \leq 2^{s_{1}} \int_{0}^{t} \frac{\mathrm{d} f(r)}{g(r)} \\
& \leq 2^{s_{1}} \int_{0}^{1} \frac{\mathrm{d} f(r)}{g(r)} \\
& =\frac{2^{s_{1}} s_{2}\left(s_{2}-s_{1}\right)}{(\log 2)^{s_{2}-s_{1}}}
\end{aligned}
$$

For the second integral, $r \geq t$ implies that

$$
\frac{1}{g(t r)}<2^{s_{1}} \frac{1}{g(t)}
$$

and hence it follows that 


$$
\int_{t}^{1} \frac{\mathrm{d} f(r)}{g(t r)}<2^{s_{1}} \int_{0}^{1} \frac{\mathrm{d} f(r)}{g(t)}=2^{s_{1}} \frac{f(1)}{g(t)} .
$$

On combining these estimates, we obtain that

$$
\begin{aligned}
-\int_{0}^{1} f(r) \mathrm{d}\left(\frac{1}{g(t r)}\right) & =\left(2^{s_{1}}-1\right) f(1) \frac{1}{g(t)}+\frac{2^{s_{1}} s_{2}\left(s_{2}-s_{1}\right)}{(\log 2)^{s_{2}-s_{1}}} \\
& \leq c_{2} \frac{1}{g(t)}
\end{aligned}
$$

for some constant $c_{2}$, as desired.

\section{Final comments}

Apart from working in higher dimensions, there are several other directions in which one could attempt to strengthen/generalize the main theorem. We concentrate on just a few of them.

The gap of uncertainty. Theorem $\mathrm{A}^{\prime}$ shows that we can not in general replace condition (77) by (9) in Theorem 2, Thus there is a gap of uncertainty associated with Theorem 2. It would be highly desirable to know whether or not condition (7) is really necessary. Namely, if $f$ and $g$ are dimension functions such that (7) is not satisfied, then does there exist a set $A \subseteq \mathbb{R}^{2}$ such that $\mathcal{H}^{f}(A)>0$ but $\mathcal{H}^{g}\left(\operatorname{proj}_{\theta} A\right)=0$ for almost all $0 \leq \theta<\pi$ ? Theorem $\mathrm{A}^{\prime}$ provides sufficient conditions on $f$ and $g$ for the existence of such a set $A$.

Brownian paths. Brownian motion sample paths, see [6, Chapter 16] for a general introduction, illustrate the sort of situation that can arise for projections of sets in $\mathbb{R}^{3}$, and which perhaps may occur in $\mathbb{R}^{2}$, though there is no direct analogue. Let $B[0,1] \subseteq \mathbb{R}^{3}$ be (random) Brownian motion path over the unit time interval. Then, almost surely, the Hausdorff dimension of $B[0,1]$ is logarithmically smaller than 2, more precisely $0<\mathcal{H}^{f}(B[0,1])<\infty$ where $f$ is the dimension function $f(r)=r^{2} \log \log (1 / r)$ (for small $r$ ), see [3]. However, the projection $\operatorname{proj}_{P}(B[0,1])$ of $B[0,1]$ onto any given plane $P$ has exactly the same distribution as a Brownian motion in the plane, which is almost surely of Hausdorff dimension 2, or precisely, $0<\mathcal{H}^{g}\left(\operatorname{proj}_{P}(B[0,1])\right)<\infty$ where $g$ is the dimension function $g(r)=r^{2} \log (1 / r) \log \log \log (1 / r)$, see [18]. This example, where the exact dimension functions of a set and of almost all its projections onto a plane can be identified, illustrates the sort of change in exact dimension that may occur under projection.

Sets with no exceptional projections. The dimension result (16) for the set of exceptional projections has been extended in various ways - see [7, 12] and 
references within. We highlight a result concerning sets $A$ for which there are no exceptional projections; that is, sets $A$ for which $E(A)=\emptyset$.

Theorem (Peres-Shmerkin). Let $A \subseteq \mathbb{R}^{2}$ be a self-similar set with dense rotations. Then

$$
\operatorname{dim} \operatorname{proj}_{\theta} A=\min \{\operatorname{dim} A, 1\} \quad \text { for all } \quad \theta \in[0, \pi) \text {. }
$$

This theorem was proved by Peres and Shmerkin [14] and subsequently generalized by Hochman and Shmerkin [8]. Now suppose $A$ is self-similar set with dense rotations and $f$ and $g$ are dimension functions as in Theorem 2 . It is natural to ask whether or not the conclusion of part (ii) of Theorem 2 is actually valid for all $\theta$ rather than just almost all $\theta \in[0, \pi)$.

Lengths of projections. It is natural to seek a finer version of part (ii) of Marstrand's theorem which gives a criterion for almost all projections of a set to have positive length. One aspect of this was investigated by Peres and Solomyak [15], who considered dimension functions $f$ such that $f(r) / r^{2}$ is decreasing for $r>0$ (a condition that holds in virtually all cases of interest). The following statement constitutes parts (i) and (ii) of their main result [15, Theorem 1.1].

Theorem (Peres-Solomyak). Let $f$ be a dimension function such that $f(r) / r^{2}$ is decreasing. Then $\int_{0}^{1} r^{-2} f(r) \mathrm{d} r<\infty$ if and only if for any Borel set $A \subseteq \mathbb{R}^{2}$ with $\mathcal{H}^{f}(A)>0$ one has that $\mathcal{L}\left(\operatorname{proj}_{\theta} A\right)>0$ for almost all $\theta \in[0, \pi)$.

Note that the integral convergence condition in the above theorem is exactly condition (7) in Theorem 9 with $g$ given by $g(r)=r$ and so $\mathcal{H}^{g}$ is Lebesgue measure $\mathcal{L}$.

Acknowledgements. SV would like to thank Julien Barral and Stéphane Seuret for organising the wonderful conference "Fractals and Related Fields III" in Porquerolles (19-25, September 2015). It was during one of the many excellent talks at this conference that the problem of Marstrand for sets of dimension zero (and hence for general dimension functions) reared its ugly head in the sparse grey matter of SV's head! SV and AZ would like to thank Henna Koivusalo for many many useful conversations and patiently listening to our ramblings.

We would like to thank the referee for carefully reading the original manuscript. Her/his comments have helped improve the clarity of the paper. 


\section{References}

[1] V. Beresnevich, V. Bernik, M. Dodson and S. L. Velani, Classical metric Diophantine approximation revisited, in Analytic Number Theory. Essays in Honour of Klaus Roth. Eds. W. Chen, T. Gowers, H. Halberstam, W.M. Schmidt and R.C. Vaughan, pp. 38-61, Cambridge University Press, 2009.

[2] H. Dickinson and S. L. Velani, Hausdorff measure and linear forms, J. Reine Angew. Math. 490 (1997), 1-36.

[3] Z. Ciesielsk and S. J. Taylor, First passage times and sojourn times for Brownian motion in space and the exact Hausdorff measure of the sample path, Trans. Amer. Math. Soc. 103 (1962), 434-450.

[4] R. O. Davis, Sets which are null or non-sigma-finite for every translation-invariant measure, Mathematika 18 (1971), 161-162.

[5] K. J. Falconer, The Geometry of Fractal Sets, Cambridge University Press, 1985.

[6] K. J. Falconer, Fractal Geometry: Mathematical Foundations and Applications, 3rd. Ed. John Wiley, 2014.

[7] K. J. Falconer, J. Fraser and X. Jin, Sixty years of fractal projections, in Fractal Geometry and Stochastics V, Eds. C. Bandt, K. J. Falconer and M. Zahle, pp. 3-25, Birkhauser, 2015.

[8] M. Hochman and P. Shmerkin, Local entropy averages and projections of fractal measures, Ann. of Math.(2) 175 (2012), 1001-1059.

[9] R. Kaufman, On the Hausdorff dimension of projections, Mathematika 15 (1968), $153-155$.

[10] J. Marstrand, Some fundamental geometrical properties of plane sets of fractional dimension, Proc. London Math. Soc.(3) 4 (1954), 257-302.

[11] P. Mattila, Geometry of Sets and Measures in Euclidean Space, Cambridge University Press, 1995.

[12] P. Mattila, Fourier Analysis and Hausdorff Dimension, Cambridge University Press, 2015.

[13] M.A. Martin and P. Mattila, $k$-dimensional regularity classifications for $s$-fractals, Trans. Amer. Math. Soc. 305 (1988), 293-315.

[14] Y. Peres and P. Shmerkin, Resonance between Cantor sets, Ergodic Theory Dynam. Systems 29 (2009), 201-221.

[15] Y. Peres and P. Solomyak, The sharp Hausdorff measure condition for length of projections, Proc. Amer. Math. Soc. 133 (2005), 3371-3379.

[16] C. A. Rogers, Hausdorff Measures, 2nd Ed. Cambridge University Press, 1998. 
[17] S. J. Taylor, On the connexion between Hausdorff measures and generalized capacity, Proc. Cambridge Philos. Soc. 57 (1961), 524-531.

[18] S. J. Taylor, The exact Hausdorff measure of the sample path for planar Brownian motion, Proc. Cambridge Philos. Soc. 60 (1964), 253-258.

Victor Beresnevich: Department of Mathematics, University of York, Heslington, York, YO10 5DD, England.

e-mail: vb8@york.ac.uk

Kenneth Falconer: Mathematical Institute, University of St Andrews, North Haugh, St Andrews, Fife, KY16 9SS, Scotland.

e-mail: kjf@st-andrews.ac.uk

Sanju L. Velani: Department of Mathematics, University of York, Heslington, York, YO10 5DD, England.

e-mail: slv3@york.ac.uk

Agamemnon Zafeiropoulos: Department of Mathematics, University of York, Heslington, York, YO10 5DD, England.

e-mail: az629@york.ac.uk 


\section{Appendix: The gap of uncertainty}

\section{David Simmons Han Yu Agamemnon Zafeiropoulos}

As discussed in Remark 3 and explicitly demonstrated in $\$ 1.2 .2$, the integral convergence condition (7) gives rise to a gap of uncertainty. It is natural to ask whether this condition is really necessary. Namely, if $f$ and $g$ are dimension functions such that (7) is not satisfied, then does there exist a set $A \subseteq \mathbb{R}^{2}$ such that $\mathcal{H}^{f}(A)>0$ but $\mathcal{H}^{g}\left(\operatorname{proj}_{\theta} A\right)=0$ for almost all $0 \leq \theta<\pi$ ? In this appendix we partially answer this question by providing sufficient conditions on $f$ and $g$ for the existence of such a set $A$. Our construction of this set is a generalization of a construction of Martin and Mattila [2], in which they proved that for every $0<s \leq 1$ there exists a set $A \subseteq \mathbb{R}^{2}$ such that $\mathcal{H}^{s}(A)>0$ but $\mathcal{H}^{s}\left(\operatorname{proj}_{\theta} A\right)=0$ for all $0 \leq \theta<\pi$. By making a careful quantitative analysis of their construction, we are able to improve their result and establish the following statement.

Theorem A. Let $f, g$ be dimension functions such that $f$ is doubling with exponent $s_{1} \leq 1$ and codoubling with exponent $s_{2}>0$, and such that

$$
g(r) \leq M f\left(r \log \left(r^{-1}\right)\right) \quad \forall 0<r<r_{0}
$$

where $r_{0}>0$ and $M>0$ are constants. Then there exists a set $A \subseteq \mathbb{R}^{2}$ with $0<\mathcal{H}^{f}(A)<\infty$ but $\mathcal{H}^{g}\left(\operatorname{proj}_{\theta} A\right)=0$ for all $0 \leq \theta<\pi$.

Recall, a dimension function $f$ is called doubling with exponent $s$ if there exist constants $\kappa>0$ and $r_{1}>0$ such that

$$
f(r \lambda) \geq \kappa \lambda^{s} f(r) \quad \forall 0<\lambda<1 \text { and } 0<r<r_{1} .
$$

Moreover, $f$ is called codoubling with exponent $s$ if there exist constants $\kappa>0$ and $r_{1}>0$ such that

$$
f(r \lambda) \leq \kappa \lambda^{s} f(r) \quad \forall 0<\lambda<1 \text { and } 0<r<r_{1} .
$$

Note that these definitions of doubling and codoubling with exponent $s$ are different from the definitions of doubling and codoubling (with constant $c>1$ ) appearing in \$1.2. It is easily seen that if a function is doubling (resp. codoubling) in the sense of (6) (resp. (10)) with constant $c>1$, then it is also doubling (resp. codoubling) in the sense of (23) (resp. (24)) with exponent $s=\log _{2} c$. (See Lemma 3 for the case of doubling functions.) However, observe that if $f$ is doubling with exponent $s \leq 1$ then it is not necessarily doubling with constant $c \leq 2$. Also, if a function is codoubling in the sense of (24) (with exponent $s>0$ ) then it is not necessarily codoubling in the sense of (10) (with constant $c>1$ ). Thus Theorem A is strictly stronger than Theorem A' appearing in Remark 3 . 
Remark 10. The assumption that the function $f$ is doubling with exponent $s \leq 1$ restricts our attention to subsets $A \subseteq \mathbb{R}^{2}$ of Hausdorff dimension at most 1 , which is expected given the nature of the problem (cf. Remark 5 ).

Remark 11. The growth condition (22) can be replaced by any condition of the form

$$
g(r) \ll f\left(r \log \left(r^{-1}\right) \log _{3}\left(r^{-1}\right) \log _{4}\left(r^{-1}\right) \cdots \log _{p}\left(r^{-1}\right)\right)
$$

where $p \geq 3$ is a positive integer and $r>0$ is sufficiently small - see Remark 14 below. Here we write $\log _{2} t=\log \log t, \log _{3} t=\log \log \log t$, etc .

Remark 12. Under the assumptions of Theorem A the integral

$$
\int_{0}^{1} \frac{\mathrm{d} f(r)}{g(r)}=\frac{f(1)}{g(1)}-\int_{0}^{1} f(r) \mathrm{d}\left(\frac{1}{g(r)}\right)
$$

diverges, hence the integral convergence condition (77) is not satisfied and in turn Theorem 2 is not violated. To see this, observe that since $f$ is doubling with exponent $s_{1} \leq 1$, we have

$$
f\left(r \log \frac{1}{r}\right) \ll f(r) \log \frac{1}{r} \quad\left(r \rightarrow 0^{+}\right) .
$$

Now since $f$ is codoubling with exponent $s_{2}>0$, we have

$$
f(r) \ll r^{s_{2}}
$$

and thus

$$
\log \frac{1}{r} \ll \log \frac{1}{f(r)} \quad\left(r \rightarrow 0^{+}\right) .
$$

Together the above estimates yield

$$
f\left(r \log \frac{1}{r}\right) \ll f(r) \log \frac{1}{f(r)} .
$$

It then follows that for any $g$ satisfying condition (22), we have

$$
\begin{aligned}
\int_{0}^{1} \frac{\mathrm{d} f(r)}{g(r)} & \gg \int_{0}^{1} \frac{\mathrm{d} f(r)}{f\left(r \log \frac{1}{r}\right)} \\
& \gg \int_{0}^{1} \frac{\mathrm{d} f(r)}{f(r) \log \frac{1}{f(r)}} \\
& =\int_{0}^{f^{-1}(1)} \frac{\mathrm{d} x}{x \log \frac{1}{x}}=\infty
\end{aligned}
$$




\section{A.1 Construction of an $f$-set}

Given a dimension function $f$, a set $A \subseteq \mathbb{R}^{n}$ is called an $f$-set if $0<\mathcal{H}^{f}(A)<\infty$. Here we present the construction of an $f$-set $A \subseteq \mathbb{R}^{2}$ for a given function $f$, which is similar to the one presented by Martin and Mattila in [2, Section 5.3] for dimension functions of the form $r \mapsto r^{s}$. In the next section, we will show that by choosing the parameters of the construction appropriately, the resulting $f$-set $A$ will satisfy $\mathcal{H}^{g}\left(\operatorname{proj}_{\theta} A\right)=0$ for all $0 \leq \theta<\pi$.

Throughout, $\left(r_{k}\right)_{k=0}^{\infty}$ is a decreasing sequence of positive real numbers tending to $0,\left(N_{k}\right)_{k=1}^{\infty}$ is a sequence of positive integers $\geq 2$, and $\left(\theta_{k}\right)_{k=1}^{\infty}$ is a sequence of angles $0 \leq \theta_{k}<\pi, k \geq 1$. The sequences $\left(r_{k}\right)_{k=0}^{\infty}$ and $\left(N_{k}\right)_{k=1}^{\infty}$ will be assumed to satisfy the inequalities

$$
a \leq N_{1} \cdots N_{k} f\left(r_{k}\right) \leq 2 a
$$

and

$$
N_{k+1} r_{k+1}<r_{k}
$$

for all $k \geq 0$, for some constant $a>0$.

Let $A_{0}$ be the closed disc of radius $r_{0}$ centered at the origin. In the first step, inside $A_{0}$ we consider $N_{1}$ subdiscs of radius $r_{1}$, denoted $C_{1}, \ldots, C_{N_{1}}$ and defined as follows: their centers are equally spaced, lying on the diameter of $A_{0}$ which forms angle $\theta_{1}$ (measured counterclockwise) with the horizontal axis, and the boundaries of first and last subdisc are tangent to the boundary of $A_{0}$. Condition (27) guarantees that these subdiscs are disjoint. Let $d_{1}=\theta_{1}$, and set

$$
A_{1}=\bigcup_{i=1}^{N_{1}} C_{i} .
$$

Now inductively assume that for some $k \geq 1$ we have defined the $\operatorname{discs} C_{i_{1} \ldots i_{k}}, 1 \leq$ $i_{j} \leq N_{j}, 1 \leq j \leq k$, each of radius $r_{k}$.

At the $(k+1)$ st step, inside each $\operatorname{disc} C_{i_{1} \ldots i_{k}}$ we consider $N_{k+1}$ subdiscs $C_{i_{1} \ldots i_{k} 1}, \ldots, C_{i_{1} \ldots i_{k} N_{k+1}}$, each of radius $r_{k+1}$, defined as follows: their centers are equally spaced along the diameter of $C_{i_{1} \ldots i_{k}}$ which forms angle $\theta_{k+1}$ with the line containing the centers of the discs of the $k$ th step, and the boundaries of the first and last subdiscs are tangent to the boundary of $C_{i_{1} \ldots i_{k}}$. Again, condition (27) guarantees that these subdiscs are disjoint. Let $d_{k+1} \equiv \theta_{1}+\ldots+\theta_{k+1}(\bmod \pi)$, so that for any disc $C_{i_{1} \ldots i_{k}}$ of $A_{k}, d_{k+1}$ is the angle between the diameter of $C_{i_{1} \ldots i_{k}}$ used to define the subdiscs of $C_{i_{1} \ldots i_{k}}$ and the horizontal axis. Set

$$
A_{k+1}=\bigcup_{\substack{1 \leq i_{j} \leq N_{j} \\(j=1, \ldots, k+1)}} C_{i_{1} \ldots i_{k+1}} .
$$


We complete the construction by setting

$$
A=\bigcap_{k=1}^{\infty} A_{k}
$$

We show that under certain conditions on $f$ and appropriate choices of the sequences $\left(r_{k}\right)_{k=0}^{\infty}$ and $\left(N_{k}\right)_{k=1}^{\infty}$, the set $A$ is an $f$-set.

Proposition A. Let $f$ be a dimension function which is doubling with exponent $s \leq 1$, and let $\left(r_{k}\right)_{k=0}^{\infty}$ be a sequence satisfying the inequalities

$$
f\left(r_{k+1}\right)<\frac{1}{4} f\left(r_{k}\right)
$$

and

$$
\frac{f\left(r_{k+1}\right)}{r_{k+1}}>3 \frac{f\left(r_{k}\right)}{r_{k}}
$$

for all $k \geq 0$. Let $\left(\theta_{k}\right)_{k=1}^{\infty}$ be any sequence of real numbers. Then the parameter $a>0$ and the sequence $\left(N_{k}\right)_{k=1}^{\infty}$ can be chosen so as to satisfy (26) and (27) for all $k \geq 0$. The resulting set $A \subseteq \mathbb{R}^{2}$ constructed as above is an $f$-set.

Proof. Let $a=f\left(r_{0}\right)$, so that (26) automatically holds when $k=0$. Now inductively assume that for some $k \geq 0$ we have chosen $N_{1}, \ldots, N_{k} \geq 2$ such that (26) holds. Since

$$
\begin{aligned}
\frac{2 a}{N_{1} \cdots N_{k} f\left(r_{k+1}\right)}-\frac{a}{N_{1} \cdots N_{k} f\left(r_{k+1}\right)} & =a \frac{f\left(r_{k}\right)}{f\left(r_{k+1}\right)} \frac{1}{N_{1} \cdots N_{k} f\left(r_{k}\right)} \\
& \stackrel{(26)}{\geq} \frac{1}{2} \frac{f\left(r_{k}\right)}{f\left(r_{k+1}\right)} \\
& \stackrel{(28)}{>} 2,
\end{aligned}
$$

the interval

$$
\left[\frac{2 a}{N_{1} \cdots N_{k} f\left(r_{k+1}\right)}, \frac{a}{N_{1} \cdots N_{k} f\left(r_{k+1}\right)}\right]
$$

contains a positive integer $N_{k+1} \geq 2$. Thus, the inequality

$$
a \leq N_{1} \cdots N_{k} N_{k+1} f\left(r_{k+1}\right) \leq 2 a
$$

is satisfied. This completes the inductive step, thus demonstrating that the sequence $\left(N_{k}\right)_{k=1}^{\infty}$ can be chosen so that (26) holds for all $k \geq 0$. 
To demonstrate (27), we note that

$$
N_{k+1} \stackrel{(30)}{\leq} \frac{2 a}{N_{1} \cdots N_{k} f\left(r_{k+1}\right)} \stackrel{(26)}{\leq} 2 \cdot \frac{f\left(r_{k}\right)}{f\left(r_{k+1}\right)} \stackrel{(29)}{<} \frac{2}{3} \cdot \frac{r_{k}}{r_{k+1}}
$$

and in particular $N_{k+1} r_{k+1}<r_{k}$.

For each $k \in \mathbb{N}$, the set $A_{k}$ is a cover of $A$ consisting of discs of radius $r_{k}$. The number of balls in this cover is $N_{1} \cdots N_{k}$, hence for $k \in \mathbb{N}$ we have

$$
\mathcal{H}_{r_{k}}^{f}(A) \leq N_{1} \cdots N_{k} f\left(r_{k}\right) \leq 2 a
$$

and thus

$$
\mathcal{H}^{f}(A)=\sup _{k>0} \mathcal{H}_{r_{k}}^{f}(A) \leq 2 a<\infty
$$

Now consider the probability measure $\mu$ supported on $A$ which is defined by assigning each of the $\operatorname{discs} C_{i_{1} \ldots i_{k}}$ of $A_{k}$ the same measure, i.e. by setting

$$
\mu\left(C_{i_{1} \ldots i_{k}}\right)=\frac{1}{N_{1} \cdots N_{k}}
$$

We claim that for all $x \in A$ and $r>0$ small enough,

$$
\mu(B(x, r)) \leq C f(r)
$$

for some constant $C>0$. By the Mass Distribution Principle (see for example [1. Lemma 3]), this will imply that $\mathcal{H}^{f}(A)>0$ and thus complete the proof.

Whenever $r, r^{\prime}$ are sufficiently small and $r<r^{\prime}$, since $f$ is doubling with exponent $s \leq 1$, for some constant $\kappa>0$ we have

$$
\frac{f(r)}{r} \geq \kappa \frac{1}{r}\left(\frac{r}{r^{\prime}}\right)^{s} f\left(r^{\prime}\right)=\kappa\left(\frac{r^{\prime}}{r}\right)^{1-s} \frac{f\left(r^{\prime}\right)}{r^{\prime}} \geq \kappa \frac{f\left(r^{\prime}\right)}{r^{\prime}},
$$

which gives

$$
\frac{f\left(r^{\prime}\right)}{r^{\prime}} \leq \kappa^{-1} \frac{f(r)}{r}
$$

Now fix $x \in A$ and $r>0$, and let $k \in \mathbb{N}$ be maximal such that $B(x, r) \cap A$ is contained in only one disc of $A_{k}$. Consider the following cases:

Case 1: $r<r_{k}$. Let $s_{k+1}$ be the common distance between any two consecutive subdiscs of $A_{k+1}$. Then by subdividing the appropriate diameter of $C_{i_{1} \ldots i_{k}}$ into intervals consisting of its intersections with discs $C_{i_{1} \ldots i_{k} j}$ as well as the gaps between them, we find that

$$
2 N_{k+1} r_{k+1}+\left(N_{k+1}-1\right) s_{k+1}=2 r_{k}
$$


Now in any sequence of $n$ consecutive subdiscs of $A_{k+1}$, the distance between the first and last subdiscs in this sequence is

$$
(n-1) s_{k+1}+(n-2) 2 r_{k+1}>(n-2)\left(s_{k+1}+2 r_{k+1}\right) .
$$

Since the diameter of $B(x, r)$ is $2 r$, if $n$ is the number of subdiscs of $A_{k+1}$ that intersect $B(x, r)$, then the distance given above must be less than $2 r$. It follows that

$$
n \leq 2+\frac{2 r}{2 r_{k+1}+s_{k+1}}
$$

On the other hand, we have

$$
\begin{aligned}
N_{k+1}\left(s_{k+1}-r_{k+1}\right) & >\left(N_{k+1}-1\right) s_{k+1}-N_{k+1} r_{k+1} \\
& \stackrel{(34)}{=} 2 r_{k}-3 N_{k+1} r_{k+1} \\
& \stackrel{(311)}{>} 0
\end{aligned}
$$

which implies that

$$
s_{k+1}>r_{k+1} .
$$

On the other hand, by the maximality of $k, B(x, r)$ intersects at least 2 discs of $A_{k+1}$, including the disc containing $x$, and thus it follows that $r>s_{k+1}$. This together with (36) implies that $r>r_{k+1}$ and so

$$
\frac{2 r}{2 r_{k+1}+s_{k+1}} \geq \frac{2}{3} \text {. }
$$

Hence,

$$
\begin{aligned}
& n \stackrel{\text { (35) }}{\leq} 4\left(\frac{2 r}{2 r_{k+1}+s_{k+1}}\right) \\
& \stackrel{\text { (34) }}{=} 8 r\left(2 r_{k+1}+2 \frac{r_{k}-N_{k+1} r_{k+1}}{N_{k+1}-1}\right)^{-1} \\
& \leq 4 r\left(r_{k+1}+\frac{r_{k}-N_{k+1} r_{k+1}}{N_{k+1}}\right)^{-1} \\
& =4 \frac{N_{k+1}}{r_{k}} r \\
& \stackrel{\text { (31) }}{\leq} 8 \frac{f\left(r_{k}\right)}{f\left(r_{k+1}\right)} \cdot \frac{r}{r_{k}} \\
& \stackrel{\text { (33) }}{\leq} \frac{8}{\kappa} \frac{f(r)}{f\left(r_{k+1}\right)}
\end{aligned}
$$


Since each subdisc of $A_{k+1}$ has measure $\frac{1}{N_{1} \cdots N_{k+1}}$, it follows that

$$
\mu(B(x, r)) \leq \frac{8}{\kappa} \frac{f(r)}{f\left(r_{k+1}\right)} \cdot \frac{1}{N_{1} \cdots N_{k+1}} \stackrel{\text { (30) }}{\leq} \frac{8}{a \kappa} f(r)
$$

Case 2: $r \geq r_{k}$. Let $C_{i_{1} \ldots i_{k}}$ be the unique disc of $A_{k}$ intersecting $B(x, r)$, which exists by the definition of $k$. Then

$$
\mu(B(x, r)) \leq \mu\left(C_{i_{1} \ldots i_{k}}\right)=\frac{1}{N_{1} \cdots N_{k}} \stackrel{\sqrt[26]{\leq}}{\leq} \frac{1}{a} f\left(r_{k}\right) \leq \frac{1}{a} f(r)
$$

where in the last inequality, we have used the fact that $f$ is increasing.

Thus in either case, (32) holds with $C=\max \left\{\frac{8}{a \kappa}, \frac{1}{a}\right\}>0$.

Remark 13. Note that Proposition A applies to any possible sequence of angles $\left(\theta_{k}\right)_{k=1}^{\infty}$, indicating that varying the sequence of angles may cause the quantity $\mathcal{H}^{f}(A)$ to change slightly but will not affect the fact that it is finite and positive. The role of the sequence $\left(\theta_{k}\right)_{k=1}^{\infty}$ will become apparent in the next section.

\section{A.2 Proof of Theorem A}

We show that if $g$ satisfies the growth condition (22) relative to $f$, the sequences $\left(r_{k}\right)_{k=0}^{\infty},\left(N_{k}\right)_{k=1}^{\infty}$ and $\left(\theta_{k}\right)_{k=1}^{\infty}$ in the aforementioned construction can be suitably selected so that the corresponding $f$-set $A \subseteq \mathbb{R}^{2}$ satisfies $\mathcal{H}^{g}\left(\operatorname{proj}_{\theta} A\right)=0$ for all $0 \leq \theta<\pi$.

First, we claim that the sequence $\left(r_{k}^{\prime}\right)_{k=k_{0}}^{\infty}$ defined by the formula

$$
r_{k}^{\prime}=(k \log k \log \log k)^{-k}
$$

satisfies (28) and (29) for all sufficiently large $k$. To prove this, we first observe that

$$
\frac{r_{k+1}^{\prime}}{r_{k}^{\prime}} \asymp \frac{1}{k \log k \log \log k} \rightarrow 0 \quad \text { as } \quad k \rightarrow \infty .
$$

On the other hand, by the doubling and codoubling hypotheses imposed on $f$, there exist constants $\kappa_{1}, \kappa_{2}>0$ such that

$$
\kappa_{1} \lambda^{s_{1}} f(r) \leq f(\lambda r) \leq \kappa_{2} \lambda^{s_{2}} f(r)
$$

for all $0<\lambda<1$ and $r>0$ sufficiently small. Since $r_{k}^{\prime}>r_{k+1}^{\prime}$ for all $k$ sufficiently large, we have that

$$
f\left(r_{k+1}^{\prime}\right) \leq \kappa_{2}\left(\frac{r_{k+1}^{\prime}}{r_{k}^{\prime}}\right)^{s_{2}} f\left(r_{k}\right)
$$


and

$$
\begin{aligned}
\frac{f\left(r_{k+1}^{\prime}\right)}{r_{k+1}^{\prime}} & \geq \kappa_{1} \frac{1}{r_{k+1}^{\prime}}\left(\frac{r_{k+1}^{\prime}}{r_{k}^{\prime}}\right)^{s_{1}} f\left(r_{k}^{\prime}\right) \\
& =\kappa_{1}\left(\frac{r_{k+1}^{\prime}}{r_{k}^{\prime}}\right)^{s_{1}-1} \frac{f\left(r_{k}^{\prime}\right)}{r_{k}^{\prime}}
\end{aligned}
$$

Thus by (38), the inequalities $(28) r_{r_{k}=r_{k}^{\prime}}$ and (29) $)_{r_{k}=r_{k}^{\prime}}$ are satisfied for all $k$ large enough. Let $k_{1} \geq k_{0}$ be chosen so that $(28)_{r_{k}=r_{k}^{\prime}}$ and $(29)_{r_{k}=r_{k}^{\prime}}$ are satisfied for all $k \geq k_{1}$.

Now consider the sequence $\left(r_{k}\right)_{k=0}^{\infty}$ defined by the formula

$$
r_{k}=r_{k+k_{1}}^{\prime}
$$

and note that (28) and (29) are satisfied for all $k \geq 0$. Thus by Proposition A, we can choose a sequence $\left(N_{k}\right)_{k=1}^{\infty}$ such that (26) and (27) hold for all $k \geq 0$. Also note that by (38) we have that

$$
\frac{r_{k+1}}{r_{k}} \asymp \frac{1}{k \log k \log \log k}
$$

Let the sequence of angles be defined by

$$
\theta_{k+1}=\frac{r_{k+1}}{r_{k}}, \quad k \geq 0
$$

Then

$$
\sum_{k=1}^{\infty} \theta_{k}=\infty .
$$

Take an arbitrary $0 \leq \theta<\pi$. Let $d_{\theta}$ denote the direction perpendicular to $L_{\theta}$, i.e. the direction of projection, $d_{\theta} \equiv \theta+\pi / 2(\bmod \pi)$. Since the series $\sum_{k=1}^{\infty} \theta_{k}$ diverges, there are infinitely many $k \in \mathbb{N}$ such that $d_{\theta}$ lies between $d_{k}$ and $d_{k+1}$. For each of these values of $k$, the angle between $d_{\theta}$ and $d_{k+1}$ is at most $\theta_{k+1}$, and thus for each disc $C_{i_{1} \ldots i_{k}}$ of $A_{k}$, the distances from the centers of all subdisc $C_{i_{1} \ldots i_{k} j}$ of $C_{i_{1} \ldots i_{k}}$ from the diameter of $C_{i_{1} \ldots i_{k}}$ in the direction $d_{\theta}$ are at most

$$
r_{k} \sin \theta_{k+1} \leq r_{k} \theta_{k+1}=r_{k+1}
$$

This is because all of these centers lie on the diameter of $C_{i_{1} \ldots i_{k}}$ in the direction $d_{k+1}$. This means that within each disc of $A_{k}$, when we project the union of the subdiscs of $A_{k+1}$ onto $L_{\theta}$ we get an interval of length at most $4 r_{k+1}$, which we can think of as the union of at most 4 intervals of length at most $r_{k+1}$. The number 
of such intervals is equal to the number of discs of $A_{k}$, that is, $N_{1} \cdots N_{k} \leq \frac{2 a}{f\left(r_{k}\right)}$.

We have shown that for infinitely many values of $k$ there is a cover of $\operatorname{proj}_{\theta} A$ which consists of $4 N_{1} \cdots N_{k}$ intervals of length at most $r_{k+1}$, hence for such $k$ we obtain that

$$
\begin{aligned}
\mathcal{H}_{r_{k+1}}^{g}\left(\operatorname{proj}_{\theta} A\right) & \leq 8 a \frac{g\left(r_{k+1}\right)}{f\left(r_{k}\right)} \\
& \leq 8 a M \frac{1}{f\left(r_{k}\right)} f\left(r_{k+1} \log \left(r_{k+1}^{-1}\right) \log \log \left(r_{k+1}^{-1}\right)\right)
\end{aligned}
$$

Now (37) implies that

$$
\begin{aligned}
\log \left(r_{k}^{-1}\right) & \asymp \log \left(r_{k}^{\prime-1}\right) \asymp k \log (k \log k \log \log k) \asymp k \log k, \\
\log \log \left(r_{k}^{-1}\right) & \asymp \log \log \left(r_{k}^{\prime-1}\right) \asymp \log (k \log k) \asymp \log k
\end{aligned}
$$

as $k \rightarrow \infty$. Combining this estimate with (39), (40), and the fact that $f$ is codoubling shows that

$$
\mathcal{H}_{r_{k+1}}^{g}\left(\operatorname{proj}_{\theta} A\right) \leq \frac{M_{1}}{(\log k)^{s_{2}}},
$$

where $M_{1}>0$ is some absolute constant. This implies that

$$
\mathcal{H}^{g}\left(\operatorname{proj}_{\theta} A\right)=\lim _{k \rightarrow \infty} \mathcal{H}_{r_{k+1}}^{g}\left(\operatorname{proj}_{\theta} A\right)=0
$$

and thereby completes the proof of Theorem A.

Remark 14. As mentioned in Remark 11, the growth condition (22) in Theorem A can be replaced by any condition of the form (25). The corresponding set in that case is constructed using the sequence $\left(r_{k}^{\prime}\right)_{k \geq k_{0}}$ defined by the formula

$$
r_{k}^{\prime}=\left(k \log k \log _{2} k \cdots \log _{p} k\right)^{-k}
$$

The proof is nearly identical and we leave it to the interested reader.

\section{References}

[1] V. Beresnevich, D. Dickinson, S. Velani, Measure theoretic laws for limsup sets, Memoirs of the American Mathematical Society 179 (2006), no. 846, 1-91.

[2] M. Martin and P. Mattila, $k$-dimensional regularity classifications for s-fractals, Trans. Amer. Math. Soc. 305 (1998), 293-315. 
David Simmons: Department of Mathematics, University of York, Heslington, York, YO10 5DD, England.

e-mail: david.simmons@york.ac.uk

Han Yu: Mathematical Institute, University of St Andrews,

North Haugh, St Andrews, Fife, KY16 9SS, Scotland.

e-mail: hy25@st-andrews.ac.uk

Agamemnon Zafeiropoulos: Department of Mathematics, University of York,

Heslington, York, YO10 5DD, England.

e-mail: az629@york.ac.uk 